\title{
Restricting carbohydrates and calories in the treatment of type 2 diabetes: a systematic review of the effectiveness of 'low-carbohydrate' interventions with differing energy levels
}

\author{
Anna P. Nicholas ${ }^{1}$ (D), Adrian Soto-Mota ${ }^{2}$, Helen Lambert ${ }^{1}$ and Adam L. Collins ${ }^{1}$ \\ ${ }^{1}$ Department of Nutritional Sciences, University of Surrey, Guildford, UK \\ ${ }^{2}$ Department of Physiology, Anatomy \& Genetics, University of Oxford, Oxford, UK
}

(Received 11 May 2021 - Final revision received 4 August 2021 - Accepted 18 August 2021)

Journal of Nutritional Science (2021), vol. 10, e76, page 1 of 15

doi:10.1017/jns.2021.67

Abstract

There are two proven dietary approaches to shift type 2 diabetes (T2D) into remission: low-energy diets (LEDs) and low-carbohydrate diets (LCDs). These approaches differ in their rationale and application yet both involve carbohydrate restriction, either as an explicit goal or as a consequence of reducing overall energy intake. The aims of this systematic review were to identify, characterise and compare existing clinical trials that utilised 'low-carbohydrate' interventions with differing energy intakes. Electronic databases CENTRAL, CINAHL, Embase, MEDLINE and Scopus were searched to identify controlled clinical trials in adults with T2D involving low-carbohydrate intake (defined as $<130 \mathrm{~g}$ carbohydrate/d) and reporting weight and glycaemic outcomes. The initial database search yielded 809 results, of which fifteen studies met the inclusion criteria. Nine out of fifteen studies utilised LCDs with moderate or unrestricted energy intake. Six trials utilised LEDs $(<1200 \mathrm{kcal} / \mathrm{d})$, with all except one incorporating meal replacements as part of a commercial weight loss programme. Interventions using both restricted and unrestricted (ad libitum) energy intakes produced clinically significant weight loss and reduction in glycated haemoglobin (HbA1c) at study endpoints. Trials that restricted energy intake were not superior to those that allowed ad libitum lowcarbohydrate feeding at 12 and 24 months. An association was observed across studies between average weight loss and reduction in HbA1c at 6, 12 and 24 months, indicating that sustained weight loss is key to T2D remission. Further research is needed to specifically ascertain the weight-independent effects of carbohydrate restriction on glycaemic control in T2D.

Key words: Diabetes: Low-carbohydrate diet: Low-energy diet: Obesity

\section{Introduction}

The prevalence of type 2 diabetes (T2D) has reached epidemic proportions and is a major global concern. According to the World Health Organization (WHO), more than 422 million people have diabetes worldwide, representing a global prevalence among adults of $8.5 \%{ }^{(1)}$. In the UK alone, over 3.9 million people are diagnosed with diabetes, $90 \%$ of which have $\mathrm{T} 2 \mathrm{D}$, and this figure is anticipated to rise to more than 5 million by $2025^{(2)}$. T2D is a major risk factor for other health conditions including cardiovascular disease, kidney failure, neuropathy and blindness ${ }^{(1)}$. It has also recently emerged as a significant risk factor for COVID-19(3).

T2D used to be considered a chronic progressive disease typically managed by escalating pharmacotherapy to maintain normoglycaemia and mitigate disease complications. However, the paradigm of treatment is changing with recognition that T2D can be put into remission ${ }^{(4)}$. Remission seems to occur up to a point, beyond which the pancreatic $\beta$-cells are unable to recover ${ }^{(5)}$. Definitions for remission vary but it is generally defined as achieving glycaemia below the diabetic range in the

Abbreviations: HbA1c, glycated haemoglobin; LCD, low-carbohydrate diets; LED, low-energy diets; T2D, type 2 diabetes; TDR, total diet replacement

* Corresponding author: Anna P. Nicholas, email annapnicholas@gmail.com 


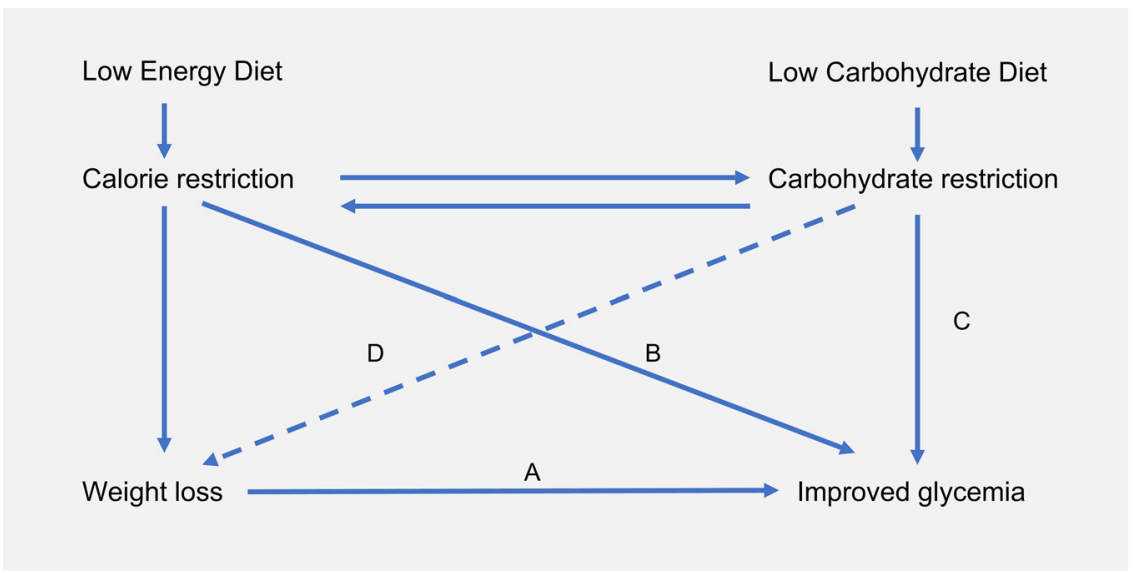

Fig. 1. Interrelationship between energy restriction, weight loss and carbohydrate restriction in improved glycaemic control: carbohydrate and energy restriction are interrelated. (A) In obese individuals with T2D, weight loss is associated with improved glycaemic control ${ }^{(10)}$. This is in accordance with the twin cycle hypothesis, whose central tenet is that excess lipids within the liver and the pancreas drive T2D pathogenesis ${ }^{(11)}$. (B) In studies of low-energy feeding, glycaemia improves within days of energy restriction, before significant weight loss has occurred ${ }^{(12)}$. (C) Carbohydrate restriction improves glycaemia by reducing postprandial glucose rises. While failed repression of gluconeogenesis and glycogenolysis are major causes of hyperglyacemia ${ }^{(13)}$, dietary carbohydrate intake is the largest driver of postprandial glucose rises. (D) Carbohydrate restriction is also associated with weight loss. This may occur as a function of spontaneous energy restriction or there may be independent effects arising from reduced insulin secretion. Whether or not carbohydrate restriction has independent effects on body weight remains a matter of contentious debate (hence depicted as dashed line) ${ }^{(14,15)}$. T2D, type 2 diabetes.

absence of antiglycaemic medications for at least 1 year ${ }^{(4)}$. The American Diabetes Association (ADA) defines three states of remission: (1) 'partial' - subdiabetic hyperglycaemia [glycated haemoglobin (HbA1c) 5·7-6.4\%] for at least 1 year; (2) 'complete' - normoglycaemic (HbA1c level $<5.7 \%$ ) for at least 1 year; (3) 'prolonged' - complete remission for at least 5 years $^{(6)}$.

There are currently two proven non-surgical ways to achieve T2D remission: low-energy diets (LEDs) and lowcarbohydrate diets $(\mathrm{LCDs})^{(7-9)}$. These two approaches focus on operating different metabolic levers: energy restriction and carbohydrate restriction. Given that both factors are interlinked (Fig. 1), it is not clear which is driving T2D remission and hence which offers the most effective interventional approach.

LEDs restrict energy intake to induce rapid weight loss ${ }^{(16)}$. They characteristically provide between 800 and $1200 \mathrm{kcal} / \mathrm{d}$ utilising either total diet replacement (TDR) or some inclusion of conventional foods as a partial diet replacement ${ }^{(17)}$. LEDs have gained attention for their use in diabetes management after the 2011 Counterpoint study which demonstrated the normalisation of $\beta$-cell function, hepatic insulin sensitivity and fasting blood glucose using an 8 week $600 \mathrm{kcal} / \mathrm{d}$ diet $^{(18)}$. In 2018, the DiRECT trial demonstrated that an intensive weight management programme using TDR could achieve T2D remission in $46 \%$ of participants after 1 year ${ }^{(8)}$.

LCDs specifically aim to restrict carbohydrate intake, either as a percentage of total energy (TE) or as an absolute intake (g/d). Definitions of what constitutes 'low carbohydrate' have been inconsistent over time and between studies but the definitions proposed by Feinman et al. ${ }^{(19)}$ are becoming more widely accepted. Specifically, this defines low carbohydrate as $<26 \%$ of TE from carbohydrates or $130 \mathrm{~g} / \mathrm{d}$ and very low-carbohydrate ketogenic diets as $<10 \%$ of TE from carbohydrates or $50 \mathrm{~g} / \mathrm{d}$.
Table 1 highlights the similarities and differences between LCDs and LEDs. Despite their differences, carbohydrate restriction is common in both: LCDs restrict carbohydrates as an explicit goal, whereas LEDs restrict carbohydrates as a consequence of achieving low-energy intake.

There is currently much interest in the use of carbohydrate restriction to treat $\mathrm{T} 2 \mathrm{D}^{(20)}$. Over the past 5 years, ten meta-analyses, based on nearly fifty randomised controlled trials (RCTs), have aimed to address the question of whether diets low in carbohydrates produce greater improvements in weight and glycaemic control compared with higher carbohydrate control diets ${ }^{(21-30)}$. The majority of these meta-analyses have found a beneficial effect from carbohydrate restriction ${ }^{(21-27)}$, and none have favoured higher carbohydrate comparators, although several studies have found no difference between diets ${ }^{(28-30)}$. Most recently, Goldenberg et al. ${ }^{(27)}$ performed a comprehensive meta-analysis of the effect of LCD $(<130 \mathrm{~g} / \mathrm{d})$ on T2D remission. They identified higher rates of diabetes remission among LCDs compared with low-fat diets at 6 months, an effect which diminished at 12 months. The authors highlighted the potential confounding role of calorie restriction but found no evidence of credible effect modification when intervention and control diets were calorically matched compared with when they were not. However, this could have been due to measurement error since calorie intakes were determined by dietary questionnaires.

Thus, the role of energy restriction in LCDs remains unclear, and no study to date has attempted to group together both LCDs and LEDs that are also 'low carbohydrate' in absolute terms. This review takes an alternative approach to the existing evidence base by recognising the commonality between these two approaches 'clamped' by carbohydrate intake. Specifically, it aims to review, characterise and compare the clinical trials that have used low-carbohydrate $(<130 \mathrm{~g} / \mathrm{d})$ approaches with different levels of energy intake. 
Table 1. Generalised similarities and differences between low carbohydrate diets and low energy diets

\begin{tabular}{|c|c|c|}
\hline & Low-energy diets & Low-carbohydrate diets \\
\hline Meal format & Usually meal replacement soups, shakes and bars & Usually food-based \\
\hline Energy restriction & Energy restricted to $\leq 1200 \mathrm{kcal} / \mathrm{d}$ & $\begin{array}{l}\text { Variable. Some allow ad libitum feeding to satiety; others include } \\
\text { moderate energy restriction ( }>1200 \mathrm{kcal} / \mathrm{d})\end{array}$ \\
\hline $\begin{array}{l}\text { Carbohydrate } \\
\text { restriction }\end{array}$ & $\begin{array}{l}\text { Variable carbohydrate content but usually at least } 50 \mathrm{~g} \\
\mathrm{CHO} / \mathrm{d}\end{array}$ & Restricted to $<130 \mathrm{~g} \mathrm{CHO} / \mathrm{d}$; VLCKD restricted to $20-50 \mathrm{~g} \mathrm{CHO} / \mathrm{d}$ \\
\hline Duration & Necessarily restricted to short periods of up to $3-5$ months & No limit on duration \\
\hline
\end{tabular}

$\mathrm{CHO}$, carbohydrate; VLCKD, very low-carbohydrate ketogenic diet.

\section{Methods}

\section{Data sources and searches}

The present systematic review was performed with reference to the Cochrane Handbook for Systematic Reviews of Interventions $^{(31)}$ and reported in accordance with the Preferred Reporting Items for Systematic Reviews and Meta-Analyses (PRISMA) statement ${ }^{(32)}$. A protocol was registered with PROSPERO in advance (CRD42020197257) ${ }^{(33)}$.

An electronic search was performed using the databases Medline, EMBASE, CINAHL, Scopus and Cochrane Central Register of Controlled Trials (CENTRAL). The search was performed on 7 July 2020, and no date limits were applied. Search terms included keywords and subject headings related to T2D, LEDs or LCDs, glycaemic outcomes and clinical trials (see Supplementary material). A manual search of reference lists of key systematic reviews and reports was also conducted to identify any additional relevant studies. Search results were independently reviewed by A.P.N. and A.S.M. and any conflicts over inclusion were resolved by discussion.

\section{Study selection}

Studies were eligible for inclusion if they were controlled trials including adults diagnosed with T2D, involving an LCD (defined as $<130 \mathrm{~g} / \mathrm{d}$ or $<26 \%$ of TE) and reporting a change in weight and glycaemic outcomes (including HbA1c, fructosamine, fasting plasma glucose and/or glycaemic variability). Non-randomised trials were eligible to allow the inclusion of trials in more ecologically valid settings, such as those utilising very low-energy weight-loss diets. Control diets that stipulated any other type of dietary intervention such as low fat, 'healthy eating' and Mediterranean, or usual diabetes care were permitted. All countries and languages were eligible. For full inclusion and exclusion criteria, see Table 2.

\section{Data extraction and quality assessment}

Data were extracted by A.P.N. Data items included: study characteristics, participant characteristics, details of the intervention (including prescribed and reported energy and macronutrient composition), dietary adherence, medication changes and outcome data for HbA1c and weight loss at 3, 6, 12 and 24 months (where available) and study endpoints. $\mathrm{HbA1c}$ only was collected as it is the most widely used marker of T2D remission, and quality of life outcomes were not reported due to lack of consistency across studies. These represent minor deviations from the protocol submitted to PROSPERO. The mean percentage weight loss from baseline and the mean absolute reduction in $\mathrm{HbA1c}$ were calculated for intervention arms. Absolute rather than relative change in HbA1c was used, since therapeutic goals are based on a threshold value and not relative reduction ${ }^{(34)}$. Risk of bias was assessed using the Cochrane Risk of Bias tool ${ }^{(35)}$.

\section{Data synthesis and analysis}

A narrative synthesis was undertaken to explore the characteristics of the included studies. HbA1c and weight loss outcome data were compared between intervention and control arms within studies at the longest duration time point.

To compare weight and HbA1c outcomes between studies, percentage weight loss and $\mathrm{HbA1c}$ change at study endpoint

Table 2. Study inclusion and exclusion criteria

Inclusion criteria

RCTs and CCTs using low $\mathrm{CHO}$ diets or very low-energy diets with $<130 \mathrm{~g} / \mathrm{d}$ or $<26 \%$ TE from $\mathrm{CHO}$ in adults ( $\geq 18$ years) with T2D Control is any other type of dietary intervention or usual care

Data from crossover trials with washout periods of $\geq 4$ weeks between interventions. In the absence of adequate washout period, data from these trials only included if able to extract relevant data for the first phase

Studies of individuals with and without T2D only if subgroup analysis available for participants with T2D

Studies reporting change in weight and markers of glucose control

Provided macronutrient goals/CHO goals as TE or $\mathrm{g} / \mathrm{d}$

\section{Exclusion criteria}

Studies that included people with other chronic diseases (except hypertension or CVD) or taking systemic corticosteroids, or had any progressive disease requiring hospital care

Studies involving participants undergoing surgery

Studies of T1D, prediabetes or gestational diabetes

Studies with enteral or parenteral feeds

Participants aged $<18$ years or pregnant/lactating women

Treatment diet poorly defined; unclear whether low $\mathrm{CHO}$ criteria were met Studies involving intermittent fasting protocols

Study duration $<1$ week

Studies based on medication co-intervention not applied to all groups 


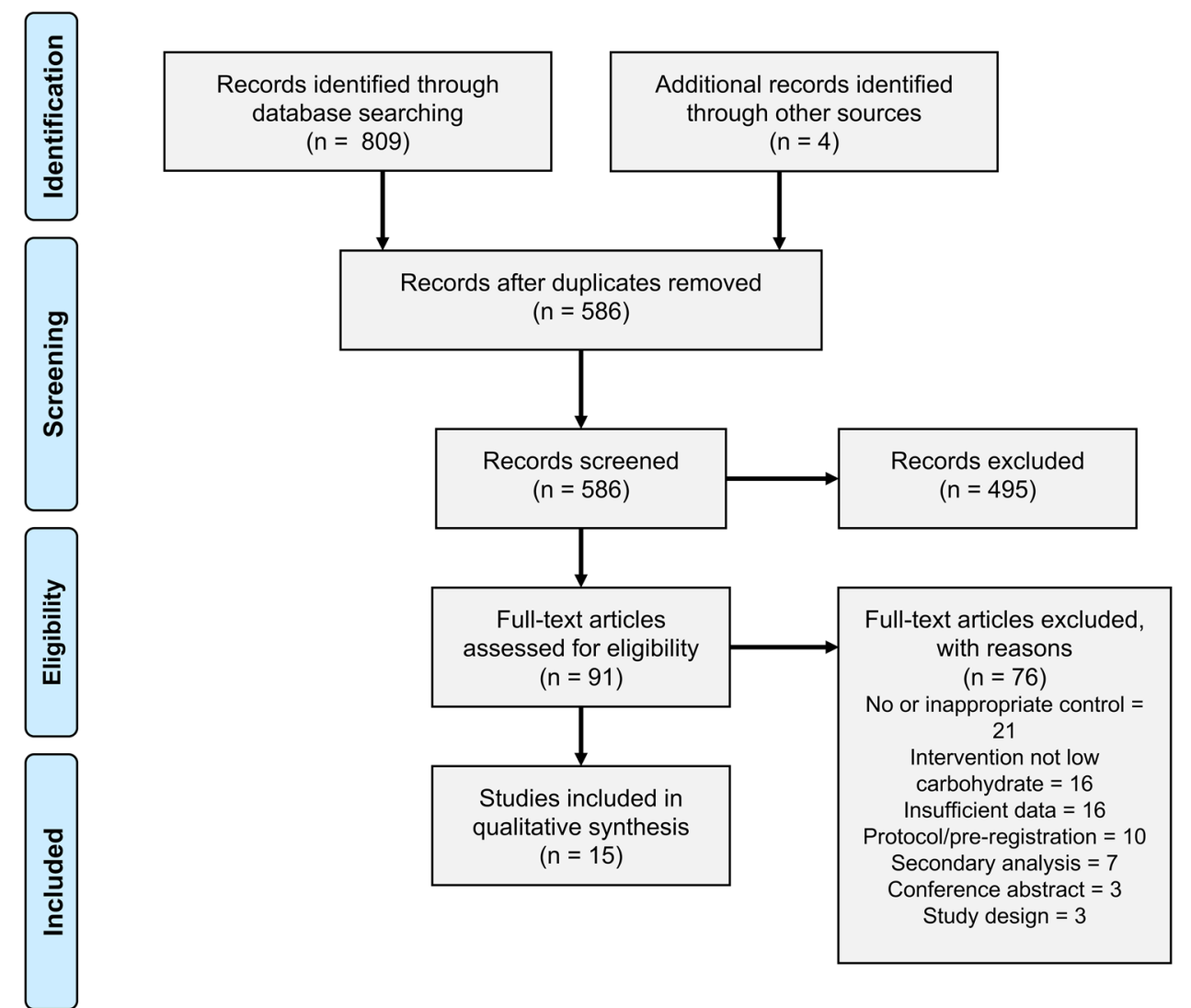

Fig. 2. Study screening and selection.

and at specific time points (3, 6, 12 and 24 months) were plotted graphically in scatter plots. Meta-analysis was not considered appropriate due to high clinical heterogeneity between studies. The association between average weight loss and $\mathrm{HbA1c}$ change was examined using correlation analysis and the computation of $R^{2}$ values in Prism 8 for OS X Version 8.4.3 $3^{(36)}$.

\section{Results}

\section{Search results}

Fig. 2 shows the selection of studies, in accordance with the PRISMA guidelines ${ }^{(32)}$. The initial database search yielded 809 studies, of which 223 were duplicates. Following title and abstract screening, ninety-one studies were retrieved for full-text screening. A total of fifteen studies met the inclusion criteria.

\section{Study characteristics}

This review yielded a highly heterogeneous set of studies that fulfilled criteria for 'low carbohydrate'. The characteristics of the fifteen controlled trials are summarised in Table 3. The publication period covered from 2006 to 2020, study duration ranged from 3 to 24 months and study sample sizes ranged from 12 to 262 participants in the intervention arm. Of the included studies, thirteen were RCTs and two were non-RCT. Of the two non-RCTs, one was randomised at the level of primary care practice rather than the participant level.

\section{Variable approaches to energy and carbohydrate restriction}

Studies were categorised into three groups based on their prescribed energy intakes: unrestricted (ad libitum feeding), moderately restricted $(1200-2000 \mathrm{kcal} / \mathrm{d})$ or severely restricted $(<1200 \mathrm{kcal} / \mathrm{d})$ (Fig. 3).

A total of seven out of fifteen studies involved traditional low or very LCDs that were food-based and did not prescribe limits on energy intake ${ }^{(37-43)}$. Two out of fifteen studies used moderate energy reduction targets ${ }^{(44,45)}$. Almost all of the studies in these groups aimed for sustained carbohydrate restriction throughout the study period. Only two were initiated by a very restrictive early phase followed by subsequent increases in carbohydrate allowance ${ }^{(38,45)}$.

Six out of the fifteen trials restricted energy intake to $<1000$ $\mathrm{kcal} / \mathrm{d}^{(46-51)}$, with all except one incorporating meal replacements as part of a stepped weight loss programme. Three studies used a 3-5 month proprietary TDR weight loss phase involving energy restriction of around $800 \mathrm{kcal} / \mathrm{d}$, followed by food reintroduction and weight loss maintenance phases ${ }^{(46,49,51)}$. In the TDR phase, carbohydrates accounted for $50-59 \%$ of TE which translated to $100-125 \mathrm{~g} / \mathrm{d}$. The TDR phase was low carbohydrate in absolute terms but the macronutrient composition of subsequent phases was unclear from the published reports. These studies did not explicitly prescribe carbohydrate restriction but rather aimed to achieve weight loss via energy restriction. There were two exceptions to this: Morris et al. ${ }^{(50)}$ used a food-based diet that was explicitly low carbohydrate ( $<26 \%$ of TE) as well as low energy 


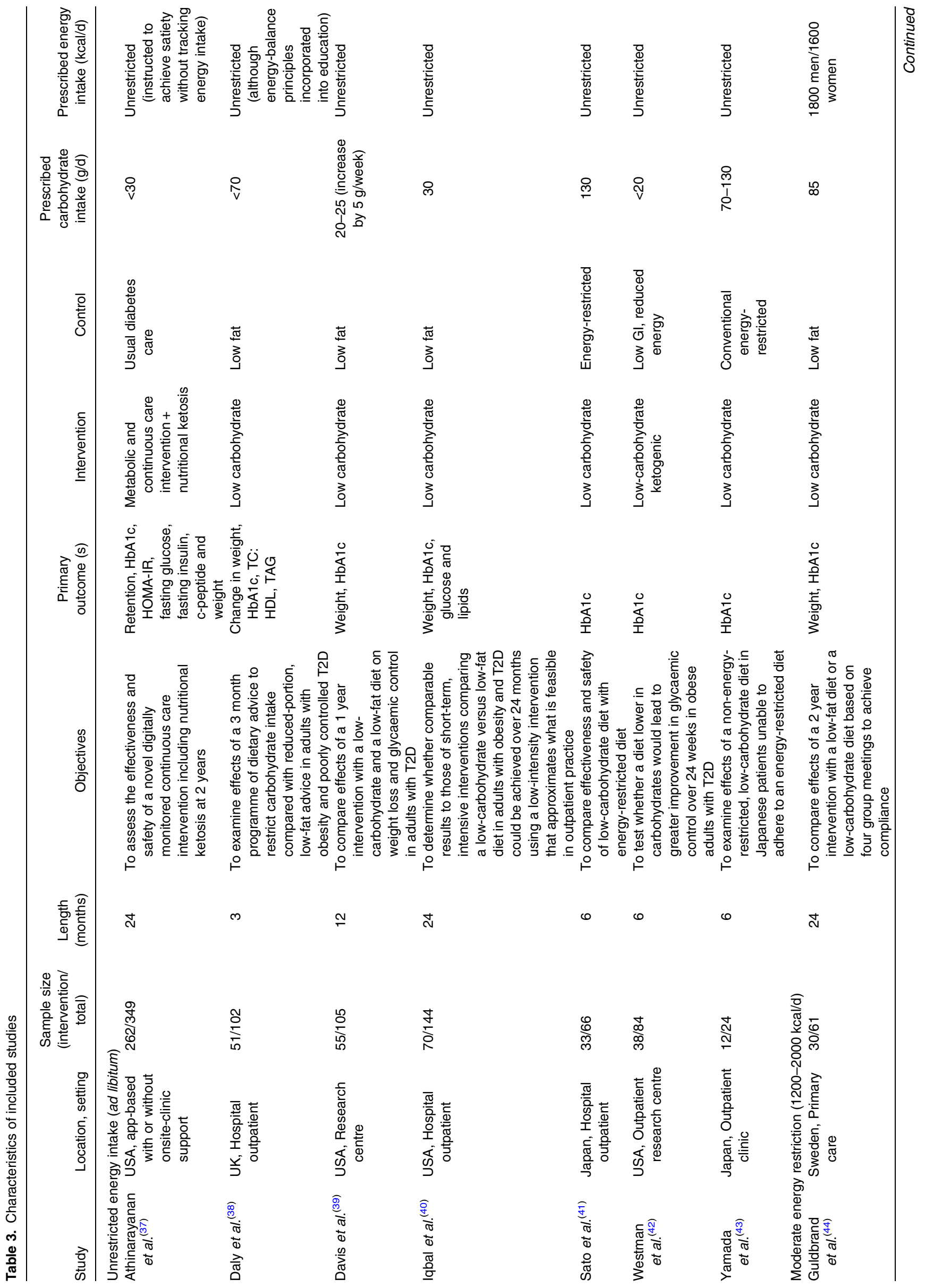




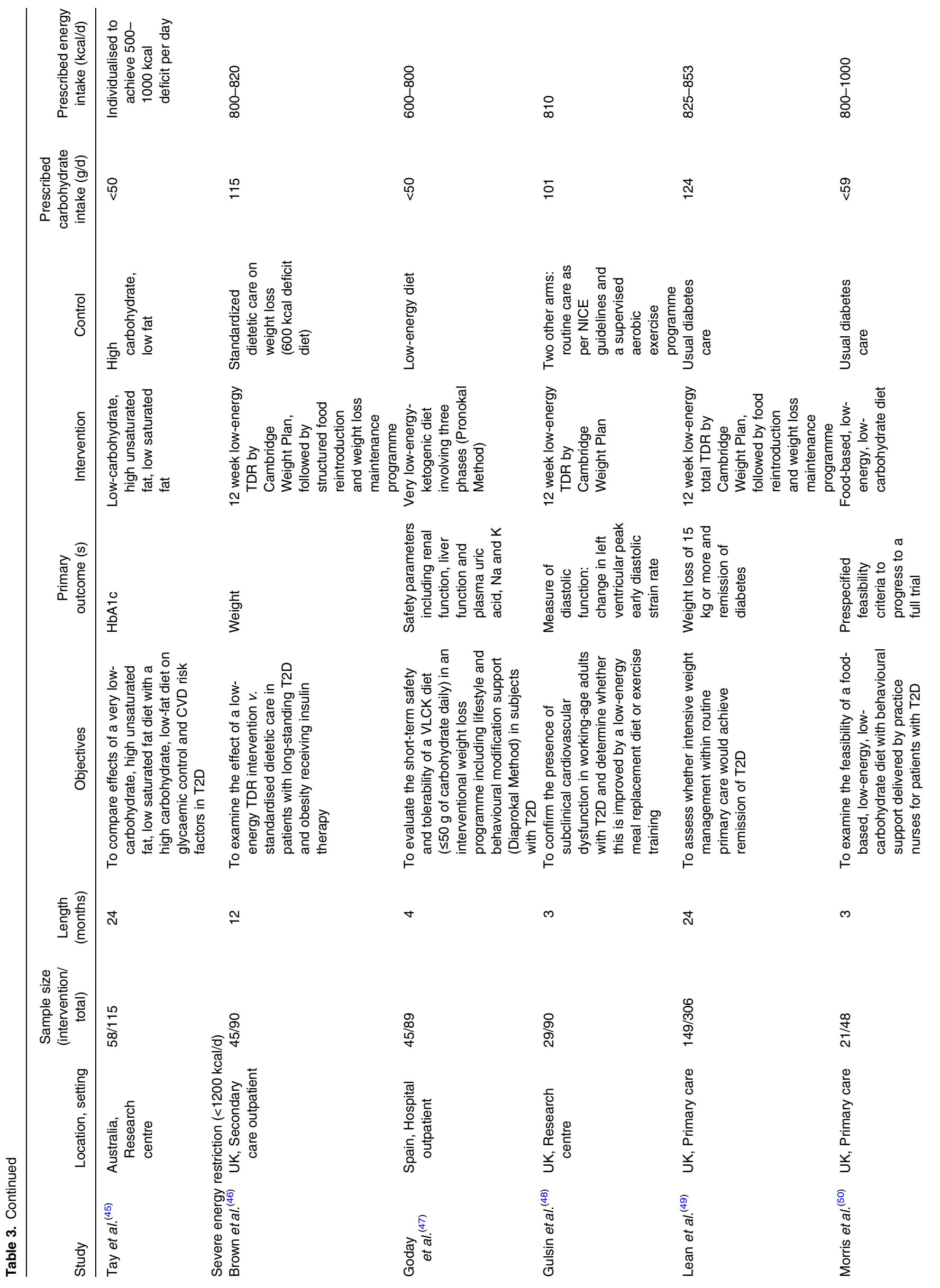




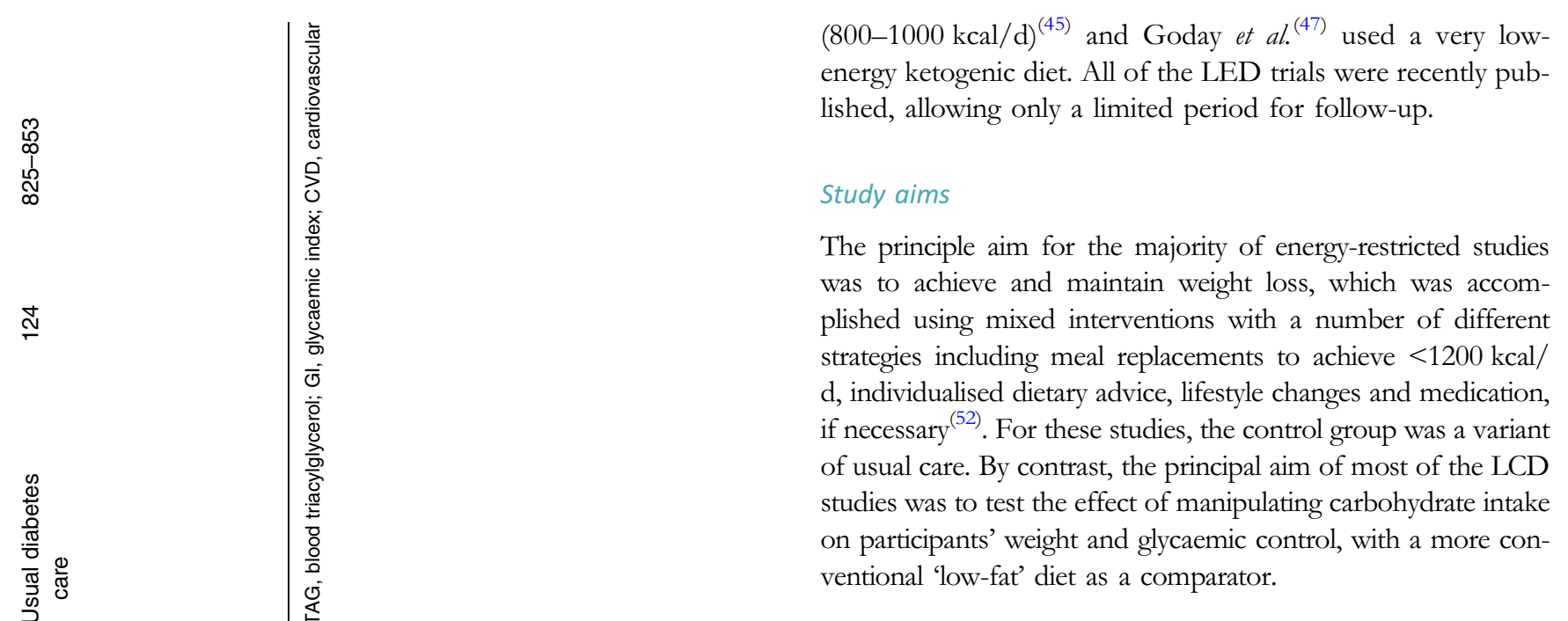

\section{Outcome measures}

Most studies' primary outcome measures related to weight and/or glycaemic control with the exception of three studies assessed cardiac function, safety parameters and full-trial feasibility criteria. All studies reported $\mathrm{HbA1c}$, with nine out of fifteen studies specifying $\mathrm{HbA1c}$ as a primary outcome. Four studies, all published in or after 2018, reported T2D 'remission' or 'reversal', although definitions varied ${ }^{(37,46,49,51)}$.

Twelve out of fifteen trials reported medication changes although methods of reporting were heterogeneous (Supplementary Table S5). All of the twelve studies that included medication changes reported a greater reduction in diabetic medications in the intervention compared with the control group. Six out of fifteen studies attempted to assess the quality of life using a range of questionnaires (Supplementary Table S1).

\section{Baseline participant characteristics}

Mean population ages ranged from 42 to 69 years, and there was a mix of ethnicities and gender ratios across studies. All studies except those conducted in Japan recruited participants who had a BMI of $>30 \mathrm{~kg} / \mathrm{m}^{2}$, with the majority having a mean BMI of $>35 \mathrm{~kg} / \mathrm{m}^{2}$. There was a wide range of average diabetes duration (2-14 years) and medication usage (Supplementary Table S2).

\section{Intervention details}

Interventions varied across studies in several ways including mode of delivery, dietary advice provided, intensity of support and utilisation of behavioural strategies to promote adherence (Supplementary Table S3). Some studies included very low-intensity interventions (involving only infrequent group sessions and dietary advice), whereas others involved more intensive input and employed a range of strategies to support dietary and lifestyle change including behaviour change techniques, intensive group support, biomarker feedback and health technologies, online support and remote care. More recent studies, published in or after 2017, involved higher intensity mixed interventions, typically employing a range of technological and behavioural support. 


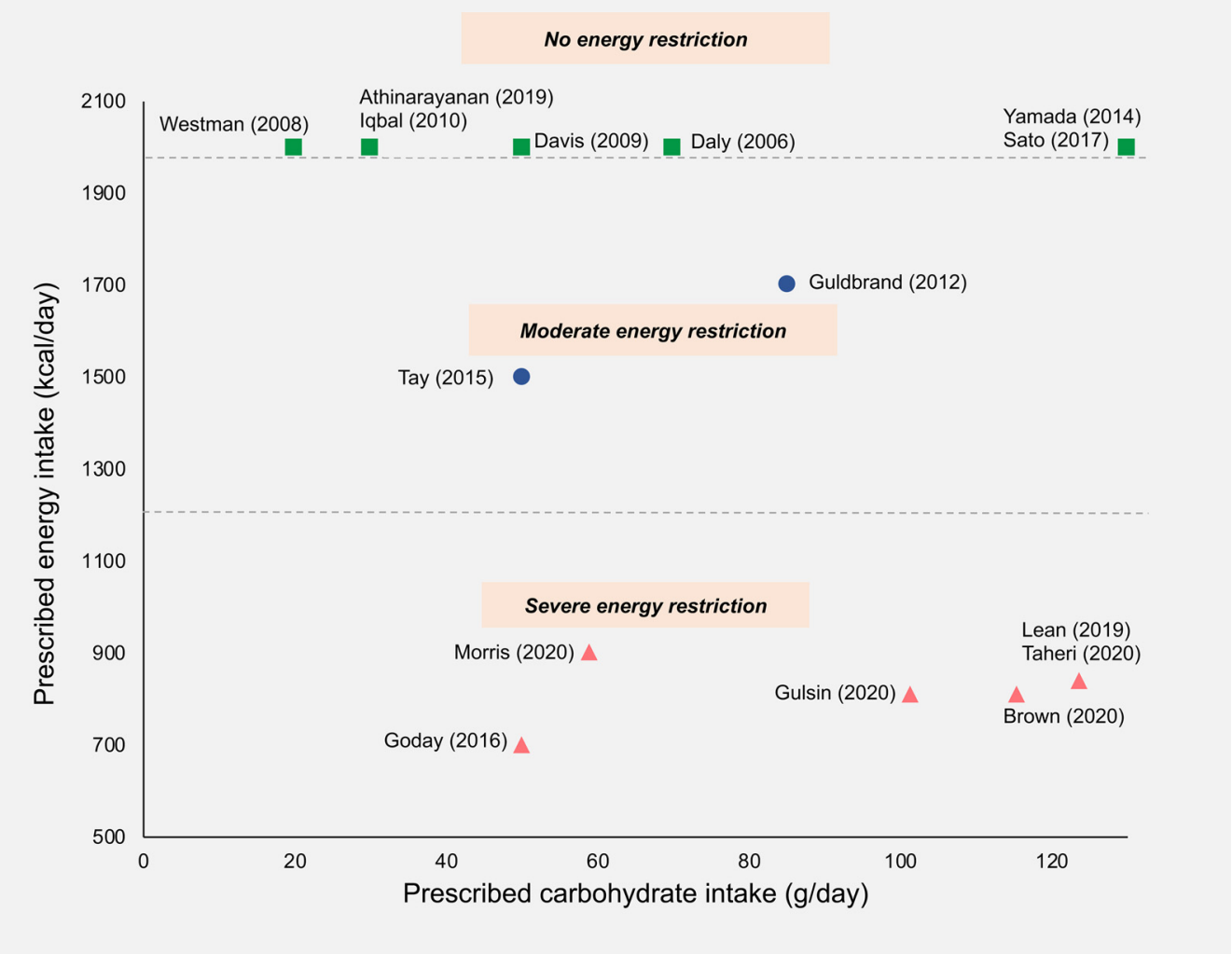

Fig. 3. Prescribed daily carbohydrate and energy intakes in included studies. Where a maximum allowance of carbohydrate or energy was prescribed, this value was used; where a range of carbohydrate or energy intakes was prescribed, the mid-point value was taken; where energy intake was unrestricted, a value of $2000 \mathrm{kcal} / \mathrm{d}$ was assigned. Squares, no energy restriction (ad libitum feeding); circles, moderate energy restriction (1200-2000 kcal/d); triangles, severe energy restriction $(<1200 \mathrm{kcal} / \mathrm{d})$.

\section{Control}

Six out of fifteen studies used usual care as a control. In five of these studies, usual care provided minimal input, so the intervention and control arms differed in a number of aspects beyond dietary change. The remaining nine studies all used a version of a low-fat, energy-restricted diet. In all but one study, energy intake was not matched between intervention and control diets. The exception was Tay et al. ${ }^{(45)}$ who used a planned isocaloric control, advising both arms to limit energy intake to achieve a 500-1000 kcal deficit per day.

\section{Dietary assessment and adherence}

The majority of studies attempted to assess dietary adherence in some way, although the methods employed varied between studies. A total of eight studies used food records $(1,3,5$ or 7-d food diaries) and two studies used self-report via questionnaires. Dietary adherence was not assessed in any of the four studies that utilised TDR (via meal replacements). Three studies used urinary or blood ketones as a marker of nutritional ketosis, one of which reported adherence based on these data ${ }^{(37)}$. Of note, in those that included participant reported carbohydrate intakes, seven out of eight studies had reported values that exceeded the prescribed carbohydrate intake by more than $10 \%$ (Supplementary Table S4).

\section{Risk of bias}

Selection bias was high for the two studies in which participants self-selected into the intervention; it was low or unclear for the thirteen trials that randomised participants between intervention arms. Six studies provided insufficient information on allocation concealment. Performance bias was judged to be high in the six studies which involved mixed interventions that differed in several aspects to the control arm, due to the nature of these trials. Performance bias was unclear for the remainder of trials due to the possible influence of participant or personnel expectations on the results. Detection bias was considered low for all studies based on the objective nature of the outcomes of interest. Attrition bias was high in three studies in which dropout rates were high or imbalanced between groups and completer analysis was used. Reporting bias of the outcomes of interest was low in all studies, since pre-specified outcomes of interest were reported. Only two studies were judged as unclear for 'other bias', due to potential baseline imbalances in confounders between the groups. The risk of bias assessment is summarised in Fig. 4 .

\section{Effectiveness of interventions}

Between-group differences. All but one study resulted in a reduction in $\mathrm{HbA1c}$ between baseline and study endpoint (Table 4). A total of eight studies demonstrated significant 


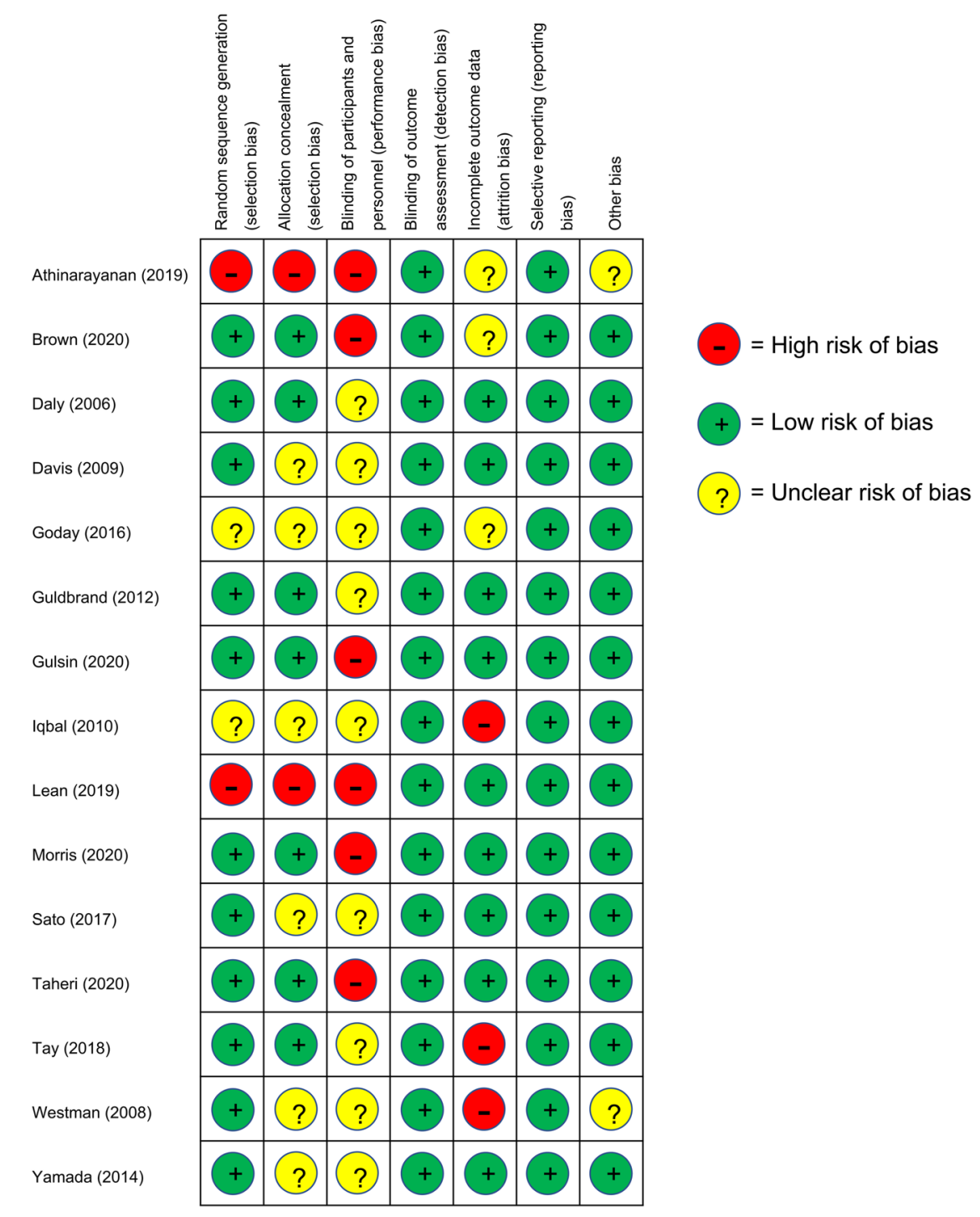

Fig. 4. Risk of bias assessment results: +, low risk of bias; ?, unclear risk of bias; -, high risk of bias.

improvements in HbA1c in the intervention arm compared with the comparator arm. All studies reported weight loss from baseline to endpoint, with nine studies showing greater weight loss in the intervention arm compared with the control group. All studies using usual diabetes care as a control arm reported significant between-group differences in weight and HbA1c. Only two of the five studies reporting data at 24 months found a difference between intervention and control groups by the end of the study ${ }^{(37,49)}$.

Weight loss and HbA1C change in intervention arms. There was a wide range of reported improvements in the intervention arms across studies in mean HbA1c change (ranging from $0 \cdot 0$ to $1.5 \%$ ) and mean percentage weight loss (ranging from only 1 to over $15 \%$ of baseline weight). Fig. 5 shows the data for all study endpoints.

Those trials that severely restricted energy all produced clinically significant weight loss of more than $5 \%$, whereas energy-unrestricted trials produced a wider range of weight and HbA1c outcomes. The non-energy-restricted studies were more numerous, published over a longer time period and involved more diverse intervention types. Two of the most effective interventions explicitly combined lowcarbohydrate and low-energy approaches ${ }^{(47,50)}$.

Fig. 6 shows the data at 12 months to facilitate comparisons between studies. The level of energy restriction did not clearly distinguish intervention efficacy. The three studies using LEDs led to a consistent and considerable mean weight loss of around $10 \%{ }^{(17,49,51)}$. The two studies reporting the largest changes at both 12 and 24 months involved LCDs with unrestricted or moderate energy restriction ${ }^{(37,45)}$. The most effective intervention at 12 and 24 months involved an ad libitum ketogenic $\operatorname{diet}^{(37)}$.

Association between weight loss and HbA1c. An association was observed between average weight loss and change in HbA1c across studies at 6, 12 and 24 months. The association was strongest at longer study lengths, as assessed 


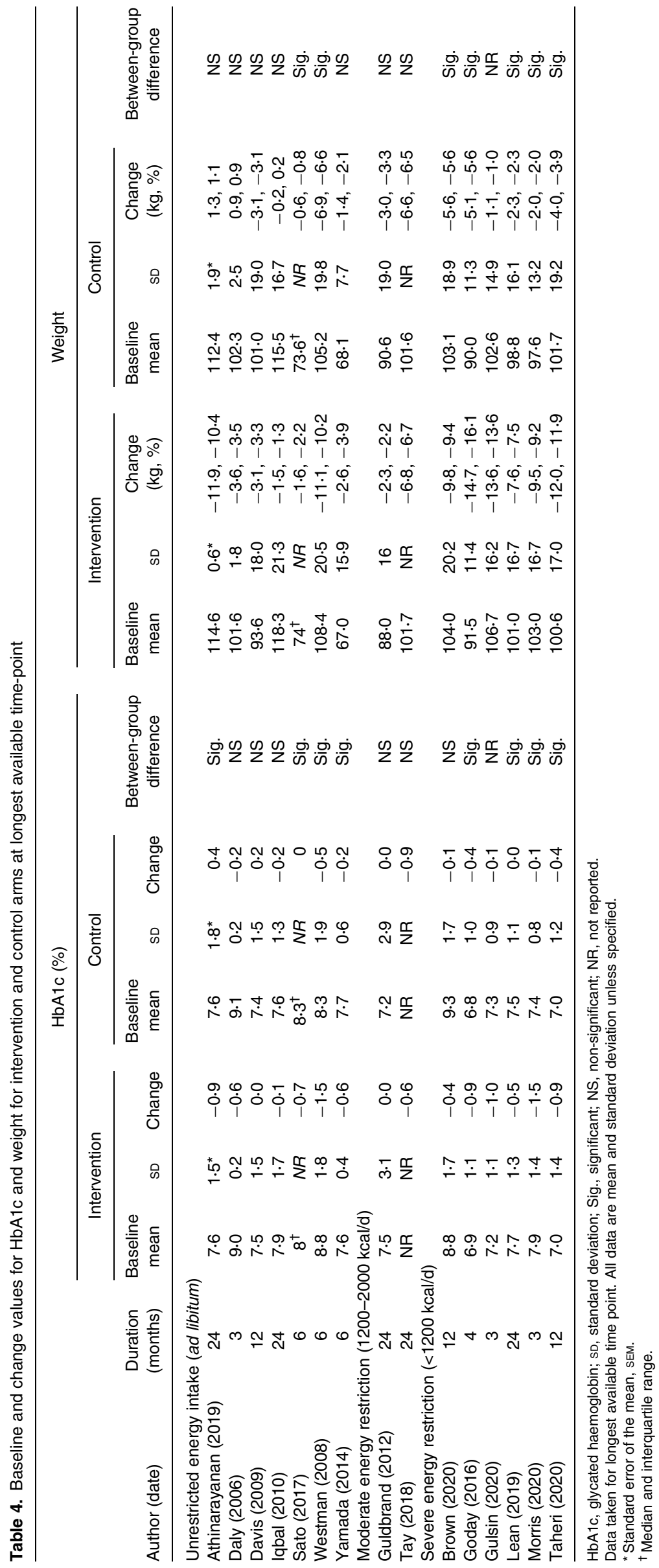




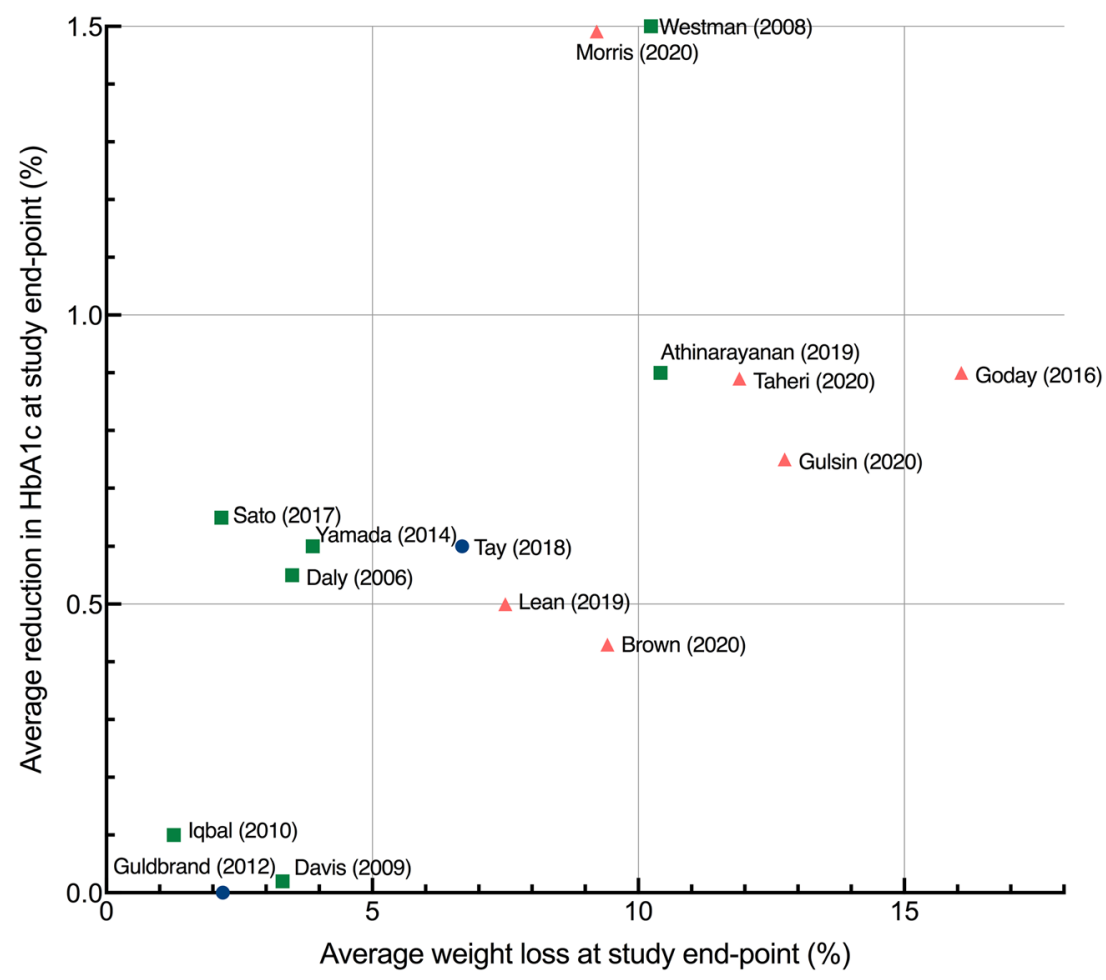

Fig. 5. Average improvement in $\mathrm{HbA} 1 \mathrm{c}$ and average percentage weight loss at study endpoints. Each point represents the mean value for a single study with the exception of Sato et al. ${ }^{(41)}$ which represents median values. Study endpoints range from 3 to 24 months. Squares, no energy restriction (ad libitum feeding); circles, moderate energy restriction (1200-2000 kcal/d); triangles, severe energy restriction (<1200 kcal/d).

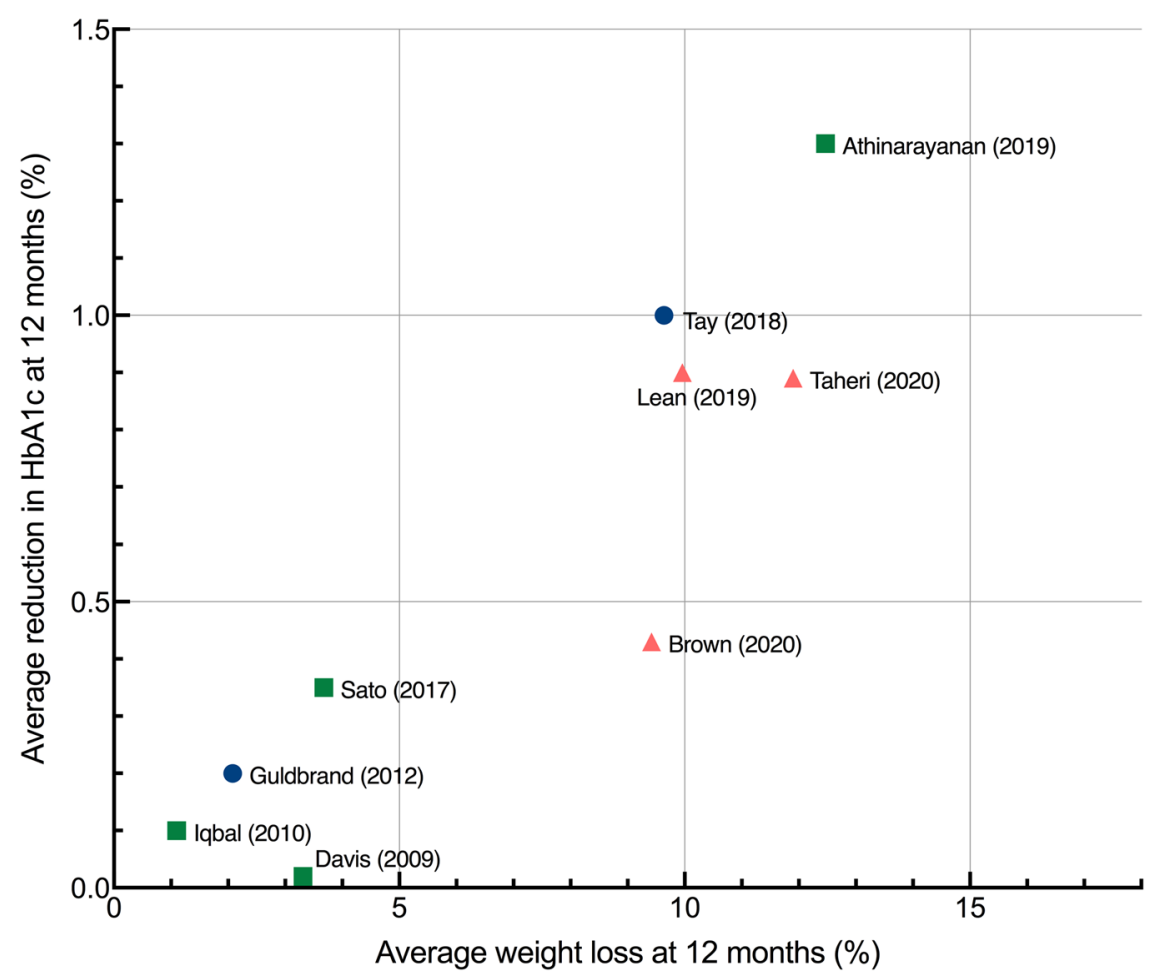

Fig. 6. Average improvement in $\mathrm{HbA} 1 \mathrm{c}$ and average percentage weight loss at 12 months each point represents the mean changes from baseline in $\mathrm{HbA1c}$ and weight for a single study, with the exception of Sato et al. ${ }^{(41)}$ which represents median values. Studies were only included if they reported data at 12 months. Squares, no energy restriction (ad libitum feeding); circles, moderate energy restriction (1200-2000 kcal/d); triangles, severe energy restriction (<1200 kcal/d). $\mathrm{HbA1c}$, haemoglobin A1C. 
by $\mathrm{R}^{2}$ : at 6 and 12 months, 84 and $82 \%$, respectively, of the variation in average $\mathrm{HbA} 1 \mathrm{c}$ change between studies could be accounted for by the variation in average weight loss; at 24 months, this increased to $91 \%$. A scatterplot summarises the results at 12 months (Fig. 6). Reductions in HbA1c were associated with the increased percentage of weight loss.

\section{Discussion}

This systematic review took a novel approach to the clinical trial evidence regarding dietary approaches to treat T2D by recognising that carbohydrate restriction is a common feature of LCDs and LEDs. Previous systematic reviews with meta-analyses have assessed the impact of higher $v$. lower carbohydrate diets ${ }^{(21-30)}$. These have shown either no effect $^{(28-30)}$ or a positive effect of carbohydrate restriction on weight loss and HbA1c $\mathrm{c}^{(21-27)}$ and have noted the role of spontaneous energy restriction in LCDs as a potential confounder.

A key strength of this review is that it only included lowcarbohydrate studies that adhered to the definitions outlined by Feinman et al. ${ }^{(19)}$. Previous systematic reviews have often included studies with higher thresholds of carbohydrate intake which limits understanding of the effects of 'true' lowcarbohydrates diets.

\section{Risk of bias}

The heterogeneity of the study designs gave rise to different risks of bias when studies were evaluated with the Cochrane Risk of Bias tool. Studies that aimed to assess the efficacy of mixed interventions (involving dietary, physical, and behavioural changes) were judged as high risk of performance bias, as they may document larger effects than those only assessing dietary changes. Additionally, two studies involved patients self-selecting the treatments they underwent which, although a valid approach for assessing efficacy, may also bias them towards reporting larger effects ${ }^{(37,49)}$. The heterogeneity of the (mostly design-inherent) sources of bias precludes head-to-head comparisons.

\section{Intervention efficacy}

This review found a range of intervention effectiveness that was not clearly distinguished by the level of energy restriction: both energy-restricted and energy-unrestricted diets were effective at 12 months, and the most effective intervention at 12 and 24 months involved an ad libitum energy-unrestricted $\operatorname{diet}^{(37)}$. This reinforces others' observations of spontaneous energy restriction in $\mathrm{LCDs}^{(53)}$ and highlights the potential efficacy of both low-carbohydrate and low-energy intervention types in the treatment of T2D.

The strength of the association between average weight loss and $\mathrm{HbA1c}$ change at 6,12 and 24 months was notable. This finding is consistent with the 'Twin Cycle Hypothesis' of T2D which proposes that T2D can be put into remission following weight loss, which reverses the accumulation of fat in the pancreatic $\beta$-cells, thereby restoring their function ${ }^{(11)}$. The potential causal relationship between weight loss and diabetes remission remains a matter of investigation ${ }^{(54,55)}$.

Regardless of causality, the strength of the association between weight loss and glycaemic markers underscores the importance of interventions that can maintain weight loss in the longer term. Weight maintenance is the most challenging area of weight management. Low-energy meal replacementbased diets are capable of producing dramatic weight loss ${ }^{(56)}$ but they are necessarily short term and weight regain is common upon cessation, especially in the absence of continued support ${ }^{(57)}$. DiRECT ${ }^{(49)}$ was the only LED trial to report data beyond 12 months and it will be of interest to see if the results achieved can be sustained over the full 5-year trial period. This review identified a greater number of clinical trials testing LCDs or very LCDs, and a correspondingly wider range of outcomes. As with DiRECT, it will be of interest to see if the results using an ad libitum ketogenic diet in the study by Athinarayanan et al. ${ }^{(37)}$ can be maintained over the full 5 -year trial period.

In line with this focus on weight loss maintenance, this review identified a trend towards interventions with greater levels of participant support through co-interventions (involving exercise, pharmacotherapy, sleep and stress-reduction), new technologies and behaviour change techniques. Previous research shows that, regardless of the modality of weight loss, participant support is important ${ }^{(58)}$, and this represents a promising trend in research.

\section{Independent role of carbohydrate restriction}

It is not clear from this review if carbohydrate restriction directly affects T2D status independent of weight loss. None of the included studies robustly measured energy intake or used an isoenergetic control, meaning the influence of spontaneous energy restriction was not controlled or accounted for. Tay et al. ${ }^{(45)}$ included a planned energy-matched high-carbohydrate control but the diet was undertaken in a free-living environment and participants in the low-carbohydrate arm reported lower energy intakes than those in the low-fat arm. Several short-term studies do indicate a weight-independent effect of carbohydrate restriction on glycaemic control ${ }^{(59-61)}$ and there are other plausible underlying mechanisms that remain under investigation $^{(62,63)}$.

The field would greatly benefit from further research to explore the potential for an independent effect of carbohydrate restriction on glycaemic control. This could be tested using a parallel-arm clinical trial comparing low-energy meal replacements with varying proportions of carbohydrates across a large enough range. Trials similar to this have been conducted using low-energy formula diets with $100 \mathrm{~g}$ (40\%) v. $162.5 \mathrm{~g}$ $(65 \%)$ carbohydrates per day and $1000 \mathrm{kcal}$ for 4 weeks ${ }^{(60)}$ and $<40 \mathrm{~g} v \cdot 65-156 \mathrm{~g} / \mathrm{d}$ for 3 weeks each (in a crossover trial) ${ }^{(59)}$. These trials have found that manipulating carbohydrates leads to differences in various markers of metabolic health. Trials using a broader range of carbohydrate intakes at fixed energy levels are needed to further explore these findings. 


\section{Implications for clinical practice}

The data in this review indicate that a major factor in T2D remission is weight loss maintenance. In clinical practice, patients would benefit from receiving information about the available options to enable them to make a fully informed individual choice, and to select for the diet and lifestyle changes that they can adhere to over the longer term, which may or may not incorporate carbohydrate restriction.

\section{Limitations}

There are several limitations in the current literature and in this review. First, presenting average weight and HbA1c outcomes of studies did not account for the underlying individual variability in weight and HbA1c outcomes.

Secondly, intervention efficacy was based solely on weight and $\mathrm{HbA1c}$ change. Some studies reported outcomes including sleep quality, anxiety and quality of life, as well as other glycaemic outcomes such as fasting blood glucose and glycaemic variability. There is also growing use and application of continuous glucose monitoring which provides measures of shortterm glycaemic control such as time in target range ${ }^{(64,65)}$. Future reviews could consider the inclusion of these and other outcomes to provide a more holistic review of the effectiveness of LEDs and LCDs in the treatment of T2D.

Thirdly, inconsistent reporting of medication adjustment across studies meant that changes in HbA1c were not considered in the context of medication changes. This may have masked differences in effect size between interventions and led to an underestimation of the positive impact of carbohydrate restriction on glycaemic control ${ }^{(22,24,25,27,29)}$. Future clinical trials would benefit from a more standardised approach to reporting medication changes to facilitate comparisons between studies.

Fourthly, due to heterogeneity in dietary assessment methods and inaccuracies associated with self-reported intakes ${ }^{(66)}$, carbohydrate and energy quantities were based on prescribed rather than actual intakes. Diet studies often suffer from poor adherence to the prescribed $\operatorname{diet}^{(67)}$ and this review also found that reported carbohydrate intake exceeded prescribed carbohydrate intake in the majority of studies. Conclusions are therefore limited to the dietary prescription of carbohydrate restriction, rather than carbohydrate restriction per se.

Finally, this review did not distinguish between ketogenic and non-ketogenic diets. Ketones have been shown to directly lower hyperglycaemia by suppressing hepatic glucose output $^{(68,69)}$. However, the role of ketosis in long-term weight loss is contentious due in part to poor adherence rates to ketogenic diets in some clinical trials ${ }^{(40)}$. This is reflected in a recent systematic review that found that LCDs were more effective than very low-carbohydrate ketogenic ones, an effect which diminished when adherence was accounted for ${ }^{(27)}$.

\section{Conclusions}

This review took a novel approach to the dietary strategies for T2D remission by recognising the commonality of carbohydrate restriction between LEDs and LCDs. It found that trials that severely restricted energy intake were not superior to those that allowed ad libitum low-carbohydrate feeding (no prescribed energy deficit) at longer study durations (12 and 24 months). However, the strong association between average weight loss and HbA1c change at 6, 12 and 24 months indicates that successful interventions for T2D are those that enable sustained weight loss in the longer term. Further studies that carefully match carbohydrate and/or energy intake between arms are needed to establish the independent roles of carbohydrate and energy restriction in T2D treatment.

\section{Supplementary material}

The supplementary material for this article can be found at https://doi.org/10.1017/jns.2021.67.

\section{Acknowledgments}

A.P.N. conceptualised the project, developed the search strategy, conducted the literature search, selected studies, extracted and interpreted the data and wrote the manuscript. A.S.M. contributed to the search strategy design, selected studies, reviewed extracted data and reviewed/edited the manuscript. H.L. and A.L.C. contributed to the project conceptualisation and search strategy design, provided final decisions on study selection and reviewed the manuscript.

There are no conflicts of interest.

\section{References}

1. World Health Organization (2020) Diabetes. https://www.who.int/ news-room/fact-sheets/detail/diabetes (accessed August 2020).

2. Diabetes UK (2020) Facts \& Figures. https://www.diabetes.org.uk/ professionals/position-statements-reports/statistics (accessed August 2020).

3. Holman N, Knighton P, Kar P, et al. (2020) Risk factors for COVID-19-related mortality in people with type 1 and type 2 diabetes in England: a population-based cohort study. Lancet Diabetes Endocrinol 8, 823-833.

4. Nagi D, Hambling C, Taylor R (2019) Remission of type 2 diabetes: a position statement from the Association of British Clinical Diabetologists $(\mathrm{ABCD})$ and the Primary Care Diabetes Society (PCDS). https://abcd.care/sites/abcd.care/files/resources/ABCDand-PCDS-final-statement-3March2019.pdf (accessed July 2020).

5. Taylor R, Al-Mrabeh A, Zhyzhneuskaya S, et al. (2018) Remission of human type 2 diabetes requires decrease in liver and pancreas fat content but is dependent upon capacity for $\beta$ cell recovery. Cell Metab 28, 547-556.e3.

6. Buse JB, Caprio S, Cefalu WT, et al. (2009) How do we define cure of diabetes? Diabetes Care 32, 2133-2135.

7. Sjöström L, Peltonen M, Jacobson P, et al. (2014) Association of bariatric surgery with long-term remission of type 2 diabetes and with microvascular and macrovascular complications. JAMA 311, 2297-2304

8. Lean ME, Leslie WS, Barnes AC, et al. (2018) Primary care-led weight management for remission of type 2 diabetes (DiRECT): an open-label, cluster-randomised trial. Lancet 391, 541-551.

9. Hallberg SJ, McKenzie AL, Williams PT, et al. (2018) Effectiveness and safety of a novel care model for the management of type 2 diabetes at 1 year: an open-label, non-randomized, controlled study. Diabetes Ther 9, 583-612. 
10. Williamson DF, Thompson TJ, Thun M, et al. (2000) Intentional weight loss and mortality among overweight individuals with diabetes. Diabetes Care 23, 1499-1504.

11. Taylor $\mathrm{R}$ (2008) Pathogenesis of type 2 diabetes: tracing the reverse route from cure to cause. Diabetologia 51, 1781-1789.

12. Lips MA, De Groot GH, Van Klinken JB, et al. (2014) Calorie restriction is a major determinant of the short-term metabolic effects of gastric bypass surgery in obese type 2 diabetic patients. Clin Endocrinol 80, 834-842.

13. Rizza RA (2010) Pathogenesis of fasting and postprandial hyperglycemia in type 2 diabetes: implications for therapy. Diabetes 59, 2697-2707.

14. Ludwig DS \& Ebbeling CB (2018) The carbohydrate-insulin model of obesity: beyond 'calories in, calories out'. JAMA Intern Med 178, 1098-1103.

15. Hall KD, Guyenet SJ \& Leibel RL (2018) The carbohydrate-insulin model of obesity is difficult to reconcile with current evidence. JAMA Intern Med 178, 1103-1105.

16. Leslie WS, Taylor R, Harris L, et al. (2017) Weight losses with low-energy formula diets in obese patients with and without type 2 diabetes: systematic review and meta-analysis. Int J Obes 41, 96-101.

17. Brown A \& Leeds AR (2019) Very low-energy and low-energy formula diets: effects on weight loss, obesity co-morbidities and type 2 diabetes remission - an update on the evidence for their use in clinical practice. Nutr Bull 44, 7-24.

18. Lim EL, Hollingsworth KG, Aribisala BS, et al. (2011) Reversal of type 2 diabetes: normalisation of beta cell function in association with decreased pancreas and liver triacylglycerol. Diabetologia 54, 2506-2514.

19. Feinman RD, Pogozelski WK, Astrup A, et al. (2015) Dietary carbohydrate restriction as the first approach in diabetes management: critical review and evidence base. Nutrition 31, 1-13.

20. Scientific Advisory Committee on Nutrition (2020) Lower carbobydrate diets for adults with type 2 diabetes. https://assets.publishing.service.gov. uk/government/uploads/system/uploads/attachment_data/file/ 989256/SACN_report_on_lower_carbohydrate_diets_for_type_2 diabetes.pdf (accessed May 2021).

21. Fan Y, Di H, Chen G, et al. (2016) Effects of low carbohydrate diets in individuals with type 2 diabetes: systematic review and meta-analysis. Int J Clin Exp Med 9, 11166-11174.

22. Snorgaard O, Poulsen GM, Andersen HK, et al. (2017) Systematic review and meta-analysis of dietary carbohydrate restriction in patients with type 2 diabetes. BMJ Open Diabetes Res Care 5, e000354. doi:10.1136/bmjdrc-2016-000354.

23. Meng Y, Bai H, Wang S, et al. (2017) Efficacy of low carbohydrate diet for type 2 diabetes mellitus management: a systematic review and meta-analysis of randomized controlled trials. Diabetes Res Clin Pract 131, 124-131.

24. Sainsbury E, Kizirian NV, Partridge SR, et al. (2018) Effect of dietary carbohydrate restriction on glycemic control in adults with diabetes: a systematic review and meta-analysis. Diabetes Res Clin Pract 139, 239-252.

25. Huntriss R, Campbell M \& Bedwell C (2018) The interpretation and effect of a low-carbohydrate diet in the management of type 2 diabetes: a systematic review and meta-analysis of randomised controlled trials. Eur J Clin Nutr 72, 311-325.

26. Van Zuuren EJ, Fedorowicz Z, Kuijpers T, et al. (2018) Effects of low-carbohydrate- compared with low-fat-diet interventions on metabolic control in people with type 2 diabetes: a systematic review including GRADE assessments. Am J Clin Nutr 108, 300-331.

27. Goldenberg JZ, Day A, Brinkworth GD, et al. (2021) Efficacy and safety of low and very low carbohydrate diets for type 2 diabetes remission: systematic review and meta-analysis of published and unpublished randomized trial data. $\mathrm{Br}$ Med J 372, m4743.

28. Schwingshackl L, Chaimani A, Hoffmann G, et al. (2018) A network meta-analysis on the comparative efficacy of different dietary approaches on glycaemic control in patients with type 2 diabetes mellitus. Eur J Epidemiol 33, 157-170.

29. Korsmo-Haugen HK, Brurberg KG, Mann J, et al. (2019) Carbohydrate quantity in the dietary management of type 2 diabetes: a systematic review and meta-analysis. Diabetes, Obes Metab 21, 15-27.

30. McArdle PD, Greenfield SM, Rilstone SK, et al. (2019) Carbohydrate restriction for glycaemic control in type 2 diabetes: a systematic review and meta-analysis. Diabet Med 36, 335-348.

31. Higgins JPT, Thomas J, Chandler J (editors), et al. (2019). Cochrane Handbook. for Systematic Reviews of Interventions Version 6.0. Cochrane. https://www.training.cochrane.org/handbook (accessed July 2020).

32. Moher D, Liberati A, Tetzlaff J, et al. (2009) Preferred reporting items for systematic reviews and meta-analyses: the PRISMA statement. Br Med J 339, b2535.

33. National Institute for Health Research. PROSPERO: international prospective register of systematic reviews. https://www.crd.york.ac.uk/ prospero/ (accessed July 2020).

34. American Diabetes Association (2010) Diagnosis and classification of diabetes mellitus. Diabetes Care 33, S62-S69.

35. Sterne J, Savović J, Page M, et al. (2019) Rob 2: A revised tool for assessing risk of bias in randomised trials. Br Med J 366, I4898.

36. GraphPad (2020) Prism 8 for Mac OS X Version 8.4.3 (471). GraphPad Software LLC.

37. Athinarayanan SJ, Adams RN, Hallberg SJ, et al. (2019) Long-term effects of a novel continuous remote care intervention including nutritional ketosis for the management of type 2 diabetes: a 2-year nonrandomized clinical trial. Front Endocrinol 10, 348.

38. Daly ME, Paisey R, Paisey R, et al. (2006) Short-term effects of severe dietary carbohydrate-restriction advice in type 2 diabetes a randomized controlled trial. Diabet Med 23, 15-20.

39. Davis NJ, Tomuta N, Schechter C, et al. (2009) Comparative study of the effects of a 1-year dietary intervention of a low-carbohydrate diet versus a low-fat diet on weight and glycemic control in type 2 diabetes. Diabetes Care 32, 1147-1152.

40. Iqbal N, Vetter ML, Moore RH, et al. (2010) Effects of a low-intensity intervention that prescribed a low-carbohydrate vs. a low-fat diet in obese, diabetic participants. Obesity 18, 1733-1738.

41. Sato J, Kanazawa A, Makita S, et al. (2017) A randomized controlled trial of $130 \mathrm{~g} /$ day low-carbohydrate diet in type 2 diabetes with poor glycemic control. Clin Nutr 36, 992-1000.

42. Westman EC, Yancy WS Jr, Mavropoulos JC, et al. (2008) The effect of a low-carbohydrate, ketogenic diet versus a low-glycemic index diet on glycemic control in type 2 diabetes mellitus. Nutr Metab 5, 1-9.

43. Yamada Y, Uchida J, Izumi H, et al. (2014) A non-calorie-restricted low-carbohydrate diet is effective as an alternative therapy for patients with type 2 diabetes. Intern Med 53, 13-19.

44. Guldbrand H, Dizdar B, Bunjaku B, et al. (2012) In type 2 diabetes, randomisation to advice to follow a low-carbohydrate diet transiently improves glycaemic control compared with advice to follow a low-fat diet producing a similar weight loss. Diabetologia 55, 2118-2127.

45. Tay J, Thompson CH, Luscombe-Marsh ND, et al. (2018) Effects of an energy-restricted low-carbohydrate, high unsaturated fat/low saturated fat diet versus a high-carbohydrate, low-fat diet in type 2 diabetes: a 2-year randomized clinical trial. Diabetes, Obes Metab 20, 858-871.

46. Brown A, Dornhorst A, McGowan B, et al. (2020) Low-energy total diet replacement intervention in patients with type 2 diabetes mellitus and obesity treated with insulin: a randomized trial. BMJ Open Diabetes Res Care 8, e001012.

47. Goday A, Bellido D, Sajoux I, et al. (2016) Short-term safety, tolerability and efficacy of a very low-calorie-ketogenic diet interventional weight loss program versus hypocaloric diet in patients with type 2 diabetes mellitus. Nutr Diabetes 6, e230.

48. Gulsin GS, Swarbrick DJ, Athithan L, et al. (2020) Effects of lowenergy diet or exercise on cardiovascular function in working-age adults with type 2 diabetes: a prospective, randomized, open-label, blinded end point trial. Diabetes Care 43, 1300-1310.

49. Lean MEJ, Leslie WS, Barnes AC, et al. (2019) Durability of a primary care-led weight-management intervention for remission of type 2 diabetes: 2-year results of the DiRECT open-label, clusterrandomised trial. Lancet Diabetes Endocrinol 7, 344-355. 
50. Morris E, Aveyard P, Dyson P, et al. (2020) A food-based, lowenergy, low-carbohydrate diet for people with type 2 diabetes in primary care: a randomized controlled feasibility trial. Diabetes Obes Metab 22, 512-520.

51. Taheri S, Zaghloul H, Chagoury O, et al. (2020) Effect of intensive lifestyle intervention on bodyweight and glycaemia in early type 2 diabetes (DIADEM-I): an open-label, parallel-group, randomised controlled trial. Lancet Diabetes Endocrinol 8, 477-489.

52. Leslie WS, Ford I, Sattar N, et al. (2016) Diabetes Remission Clinical Trial (DiRECT): protocol for a cluster randomised controlled trial in primary care. BMC Fam Pract 17, 20.

53. Johnstone A, Horgan G, Murison S, et al. (2008) Effects of a highprotein ketogenic diet on hunger, appetite, and weight loss in obese men feeding ad libitum. Am J Clin Nutr 87, 44-55.

54. Taylor R \& Holman RR (2015) Normal weight individuals who develop type 2 diabetes: the personal fat threshold. Clin Sci 128, 405-410.

55. Al-Mrabeh A (2020) Pathogenesis and remission of type 2 diabetes. Cardiovasc Endocrinol Metab 9, 132-142.

56. Rehackova L, Arnott B, Araujo-Soares V, et al. (2016) Efficacy and acceptability of very low energy diets in overweight and obese people with type 2 diabetes mellitus: a systematic review with meta-analyses. Diabet Med 33, 580-591.

57. Brown A, Dornhorst A, McGowan B, et al. (2020) Low-energy total diet replacement intervention in patients with type 2 diabetes mellitus and obesity treated with insulin: a randomized trial. BMJ Open Diabetes Res Care 8, e001012.

58. Hall KD, Kahan S \& Diseases K (2018) Maintenance of lost weight and long-term management of obesity. Med Clin North Am 102, 183-197.

59. Gumbiner B, Wendel JA \& McDermott MP (1996) Effects of diet composition and ketosis on glycemia during very-low-energy-diet therapy in obese patients with non-insulin-dependent diabetes mellitus. Am J Clin Nutr 63, 110-115.

60. Miyashita Y, Koide N, Ohtsuka M, et al. (2004) Beneficial effect of low carbohydrate in low calorie diets on visceral fat reduction in type 2 diabetic patients with obesity. Diabetes Res Clin Pract 65, 235-241.

61. Hyde PN, Sapper TN, Crabtree CD, et al. (2019) Dietary carbohydrate restriction improves metabolic syndrome independent of weight loss. JCI Insight 4, e128308.

62. Soto-Mota A (2018) The mechanism whereby an exogenous ketone drink lowers blood glucose. ISRCTN. doi:10.1186/ISRCTN16169021.

63. Soto-Mota A (2018) Safety of twenty-eight-day consumption of $\Delta G \AA$ in healthy adults and type 2 diabetes patients. ISRCTN. doi:10.1186/ISRCTN12401551

64. Chehregosha H, Khamseh ME, Malek M, et al. (2019) A view beyond HbA1c: role of continuous glucose monitoring. Diabetes Therapy 10, 853-863.

65. Krhač M \& Lovrenčić MV (2019) Update on biomarkers of glycemic control. World J Diabetes 10, 1-15.

66. Thompson FE \& Subar AF (2012) Dietary assessment methodology. In Nutrition in the Prevention and Treatment of Disease 3rd Edition [A Coulston, C Boushey \& M Ferruzzi Eds.]. San Diego: Academic Press.

67. Gibson AA \& Sainsbury A (2017) Strategies to improve adherence to dietary weight loss interventions in research and real-world settings. Behav Sci 7, 44.

68. Ari C, Murdun C, Koutnik AP, et al. (2019) Exogenous ketones lower blood glucose level in rested and exercised rodent models. Nutrients 11, 2330.

69. Stubbs BJ, Cox PJ, Evans RD, et al. (2017) On the metabolism of exogenous ketones in humans. Front Physiol 8, 848. 